\title{
LA PROGRAMACIÓN DE ABASTECIMIENTO Y SU INCIDENCIA EN LA GESTIÓN LOGÍSTICA EN LA UNIVERSIDAD NACIONAL JORGE BASADRE GROHMANN
}

\author{
SUPPLY PROGRAM AND ITS EFFECT IN LOGISTIC MANAGEMENT IN \\ JORGE BASADRE GROHMANN NATIONAL UNIVERSITY
}

\author{
${ }^{1}$ Felipe Yony Gómez Cáceres
}

\begin{abstract}
RESUMEN
La presente investigación busca estudiar y analizar los procesos técnicos de la programación de abastecimiento, a fin de establecer y determinar su incidencia en la gestión de logística de la Universidad Nacional Jorge Basadre Grohmann de Tacna. La programación de abastecimiento es la base del ordenamiento que debe existir en el proceso logistico; también es el punto de partida, apenas se tenga necesidad de algún material, por lógica debemos determinar la cantidad que necesitamos y para cuándo lo requerimos. Sin la programación de abastecimiento, la función de logística simplemente no cumplirá con su objetivo y las personas que trabajan en ella, se convertirán en "mandaderos", "regularizadores", "notarios"; pues todos los pedidos serán siempre "urgentes" para "ayer". Para alcanzar el objetivo señalado, se realizó un tipo de investigación básica, de carácter descriptiva correlacional, en el que participaron todo el potencial humano que labora en la Oficina de Logistica y Servicios de la UNJBG. Se determinó y se concluyó que la gestión de logística en la UNJBG no es eficiente, por lo cual, se recomienda realizar y aplicar la programación de abastecimiento, a fin de superar y mejorar la gestión de logistica.
\end{abstract}

Palabras Clave: programación de abastecimiento, gestión de logistica.

\section{ABSTRACT}

This research sought to study and analyze the technical processes of supply program, in order to establish and determine its impact on the management logistics management in Jorge Basadre Grohmann National University of Tacna. The supply program is the base of the system that must exist in the logistics process; it is also the starting point when some material is needed, the amount needed and when it is required logically must be determined. Without the supply program the logistics function will simply not meet its goal and the people working in it will become "messengers", "regularizadores", "notaries"; all orders will always be "urgent" for "yesterday". To achieve the stated objective, a kind of basic, co relational and descriptive research was developed where all human potential working in the Logistics and Services office in the Jorge Basadre Grohmann National University participated (UNJBG). It was determined and found that logistics management in UNJBG is not efficient, so it is recommended to develop and apply a supply program, in order to overcome and improve logistics management.

Key Words: supply program, logistic management.

\section{INTRODUCCIÓN}

Las entidades públicas son organismos en constante cambio, su razón de existir se fundamenta en satisfacer los objetivos del gobierno (Central, Regional y Local) y las necesidades de la colectividad. Se crean, desarrollan, transforman y extinguen, de acuerdo con estos requerimientos y demandas tanto de la comunidad como del gobierno. Las entidades públicas como la Universidad Nacional Jorge Basadre Grohmann se crean por Ley de la República; la implementación administrativa de este mandato es el proceso que se denomina organización, proceso en el cual se diseña y pone en funcionamiento el aparato administrativo que orientará a cumplir con los fines y objetivos de la entidad.

En la administración pública se distinguen tres tipos de sistemas de interrelación dinámica: sistema de decisión política, sistemas de gestión y sistemas auxiliares, este último se ha generalizado o se conoce como sistemas administrativos, referidos a los elementos dinamizadores de la administración pública en general y de cada una de las entidades que la integran. Estos sistemas comprenden, tanto órganos centrales como periféricos, los primeros son los rectores y los segundos, ubicados en cada una de las entidades como las universidades públicas constituyen, los ejecutores.

Master of Sciencie con mención en Gestión Empresarial, Lieenciado en Administración, Jefe de la Oficina General de Planificación de la UNJBG. Facultad de Ciencias Juridicas y Empresariales de la Universidad Nacional Jorge Basadre Grohmann. Tacna-Peri. 
A los sistemas administrativos se los clasificó en sistemas de recursos: (personal, financiero y abastecimiento) y sistemas de equilibrio (planificación, presupuesto, control y racionalización). A la luz de esta concepción se desarrollaron $y$ fortalecieron los actuales sistemas administrativos, entre los cuales está el sistema de abastecimiento y sus subsistemas y procesos técnicos, como es la programación de abastecimiento.

El sistema de abastecimiento en la administración del Estado se orienta a suministrar los bienes materiales y servicios no personales denominados insumos para la operatividad gubernativa de los servicios públicos o producción de bienes materiales y servicios, a través de las distintas organizaciones empresariales que constituyen el aparato estatal.

La programación de abastecimiento es la base fundamental del ordenamiento que debe existir en el proceso logístico; también es el punto de partida. Apenas tenemos necesidad de algún material, por lógica se determina la cantidad que necesitamos y para cuándo lo requerimos, comparando con el objetivo logístico, la programación nos va determinar la oportunidad y la cantidad de los bienes y servicios que necesita una entidad.

El objetivo es estudiar y analizar cada uno de los procesos técnicos de la programación de abastecimiento; con la finalidad de establecer y determinar su incidencia en la gestión de logística de la UNJBG.

\section{Estado de Arte}

Se desconoce de investigaciones realizadas en nuestra región sobre el tema específico; lo que se ha encontrado y existe son antecedentes indirectos, entre estos trabajos señalamos las ejecutadas por la Dirección Nacional de Abastecimiento de la ex INAP, Institución Rector del Sistema que desarrolló las funciones normativas y procesos técnicos que deberían cumplir los organismos del Estado.

La referida institución ha realizado estudios generales referentes a la programación de abastecimiento como una de las etapas fundamentales del sistema logístico, pero no ha realizado estudios por etapas o por subsistemas.

\subsection{Enfoques y evolución histórica de la logística}

Logística es un término muy antiguo, usado por los griegos para denominar así a un sistema para resolución de problemas matemáticos. Militarmente fue usado por primera vez en Francia, alrededor de 1880, en que se creó un batallón de logística. A través del tiempo, se le encargó a la logística militar, la responsabilidad del movimiento, transporte $y$ aprovisionamiento de tropas.

La logística tuvo el inicio de su gran desarrollo en la segunda guerra mundial, en que se generalizó a todas las empresas e instituciones.

En este siglo XXI la logística es ya un proceso de búsqueda, un proceso de reconstrucción de la fabricación segmentada y deslocalizada y se está imbricando en un proceso de marketing, comercialización y ventas como soporte clave del nivel de servicio a clientes y estrategia extrema para agregar valor al producto para satisfacción y fidelización del consumidor final.

En la actualidad, la logística tiene un gran protagonismo, la cual está avalada por el crecimiento de la distribución y la competencia a nivel internacional. Es más, la logística está extendiendo su campo a empresas que, en lugar de productos materiales, están proporcionado servicios, teniendo en cuenta que, en la actualidad y cada vez más, la economía se apoya en este sector, además del industrial (Casanovas, 2003).

\subsection{Enfoque, doctrina y evolución histórica del sistema de abastecimiento}

El sistema de abastecimiento se constituye en la actualidad en el apoyo estratégico para alcanzar las metas previstas en la programación presupuestaria a fin de atender con eficiencia y eficacia las siempre urgentes e inagotables necesidades de bienes y servicios que requieren las entidades, en el marco de la gestión Institucional.

La concepción y doctrina del abastecimiento han evolucionado en la medida que el funcionario y servidores del Estado se han ido formando en el trabajo para ofrecer mejores servicios públicos.

Como antecedentes a la creación del sistema de abastecimiento, podemos mencionar que el 13 de junio de 1969, mediante el Decreto Supremo Nº86-HA, se creó la Comisión Técnica de Trabajo del sistema de compras y adquisiciones, como parte de la Comisión de Reforma de la Administración Pública.

Posteriormente, el equipo de trabajo $\mathrm{N}^{\circ} 03$ del Sistema de Abastecimiento, como parte de la Comisión de Reforma de Administración Pública creada mediante Decreto Supremo N ${ }^{\circ}$ 008-73-PM de fecha 11 de mayo de 1973, estructura las bases del Abastecimiento, para su creación posterior como sistema auxiliar, la misma que conocemos en la actualidad como sistema administrativo de abastecimiento,

El sistema de abastecimiento fue instituido por Decreto Ley $\mathrm{N}^{\circ} 22056$ y tuvo su órgano rector en la Dirección Nacional de Abastecimiento del ex Instituto Nacional de Administración Pública - INAP, organismo que fue declarado en disolución por mandato de la Ley $\mathrm{N}^{\circ}$ 26507.

Hasta el 29 de setiembre de 1998, el Sistema de Abastecimiento estaba normado por el "Reglamento Único para el Suministro de Bienes y Prestación de Servicios No Personales para el Sector Público" RUA, aprobado por Decreto Supremo No 065-85-PCM, el "Reglamento Único de Licitaciones y Contratos de Obras Públicas"- RULCOP aprobado por Decreto Supremo $\mathrm{N}^{\circ}$ 034-80-VC y el Reglamento de Actividades de Consultoría- REGAC; dispositivos legales que son derogados por la Ley $\mathrm{N}^{\circ} 26850$ Ley de Contrataciones y Adquisiciones del Estado, que entra en vigencia a partir del 29 de setiembre del 1998.

Mediante Leyes números 27070, 27148 y 27330 se efectuaron modificaciones a la Ley $\mathrm{N}^{\circ} 26850$, lo cual hizo necesaria la promulgación del Decreto Supremo $N^{\circ} 012$ 2001-PCM que aprueba el Texto Único Ordenado de la Ley de Contrataciones y Adquisiciones del Estado; dispositivos legales que entran en vigencia a partir del 15 de marzo del 2001 .

En 2004 se modifican y se promulga el nuevo texto ordenado de la Ley de Contrataciones y Adquisiciones del 
Estado mediante el Decreto Supremo No 083-2004 PCM, y su Reglamento el Decreto Supremo No 084-2004-PCM.

El Poder Ejecutivo el 03 de junio del 2008 ha promulgado el Decreto Legislativo $\mathrm{N}^{\circ} 1017$ Ley de Contrataciones del Estado y se publicó en el Diario Oficial El Peruano, el 04 de junio del mismo año. Con esta norma se derogó la Ley $\mathrm{N}^{\circ} 26850$ y posteriormente, dentro de los plazos establecidos en la Ley, se aprobó su Reglamento mediante el Decreto Supremo $N^{\circ} 084-2008-E F$, las mismas que se encuentran vigentes a la fecha.

Anaya (2007) señala que hoy en día, no se puede concebir una empresa como un ente aislado moviéndose como una ameba dentro del turbulento mundo de la actividad empresarial, sino tiene que integrarse a través de cadenas de distribución logistica.

Gutiérrez (1998) señala que uno de los objetivos de la gestión de compras dentro de la logística es la Previsión de necesidades, el proceso tiene su origen en el plan de ventas y en el de producción. Las previsiones de ventas se efectúan mediante una estimación basada en el comportamiento que se produjo en el pasado ajustándola a través de los cambios en las expectativas de futuro.

Carter (2003) señala que conforme las compañías se esfuerzan por ser competitivas a nivel mundial, la administración eficaz de compras y materiales se ha convertido en un campo de gran interés e importancia, y desempeña un papel crítico en la reestructuración y restauración de las industrias que intentan ganar o recuperar, una posición mundial. Asimismo, añade que los líderes de la industria reconocen en general, que para alcanzar una competitividad global, se necesita una administración eficiente de los recursos productivos y materiales de la organización.

Kolb (1998) señala que el sistema logístico de la empresa está constituido por el conjunto de medios de producción, de transporte, de manipulación y de almacenamiento aplicados para hacer pasar los productos del estado de materias primas almacenadas en manos de los proveedores al de productos elaborados entregados a los clientes. El sistema logístico asegura, pues tres grandes funciones: aprovisionamiento, producción y distribución fisica.

Asimismo, añade Kolb, que la logística agrupa todos los métodos de organización y de gestión aplicados al diseño, funcionamiento y control del sistema logístico. Se ocupa de la elección de los medios y de los métodos en el campo de los transportes, de la manipulación y del almacenamiento, y de la elaboración de las previsiones, planes y programas de aprovisionamiento, producción y distribución.

El autor señala también que el mejor medio de ascgurar una planificación eficaz de la salida de los flujos de materias y de productos es, $\sin$ duda, una completa integración, al nivel funcional de las responsabilidades en materia de logística y una mejor evaluación de la incidencia de los objetivos comerciales sobre el costo de aplicación del sistema logístico.

Martínez (2001) senala que la misión general del aprovisionamiento consiste en obtener del exterior a la empresa, los materiales, productos $y / 0$ servicios que necesite para su funcionamiento en las cantidades y plazos establecidos, con los niveles de calidad necesarios y al menor precio que permita el mercado.

Añade el autor, que estos objetivos serian válidos para cualquier tipo de empresa ya sea de tipo industrial o comercial y, en función de la estrategia competitiva de cada empresa, se pondrá el acento en los aspectos cualitativos o cuantitativos.

El Sistema de Abastecimiento fue instituido por Decreto Ley $\mathrm{N}^{\circ} 22056$ y tuvo su órgano Rector en la Dirección Nacional de Abastecimiento del ex Instituto Nacional de la Administración Pública-INAP, organismo que fue declarado en disolución por mandato de la Ley $\mathrm{N}^{\circ}$ 26507 , sin embargo, como sistema sigue funcionando en todas las dependencias del Estado.

Desde el punto de vista de la actividad pública, el concepto de la palabra logística está enmarcada en relación con la naturaleza y las funciones que tienen que cumplir las Entidades Públicas, puesto que ellos tienen como propósito principal brindar un "servicio público a la comunidad en general", sin ánimo de lucrar u obtener algún beneficio propio.

La actividad Logística de las citadas entidades se orienta básicamente a la programación, adquisición, almacenamiento y distribución de los bienes para su uso (activos fijos) y/o consumo (bienes fungibles), con el objeto de que ellas desarrollen sus actividades en condiciones normales, que les permita alcanzar sus metas y objetivos.

Asimismo, comprende en forma complementaria la prestación de los servicios que toda Entidad Pública requiere para el cumplimiento de su función (servicios de agua, energía eléctrica, teléfono, mantenimiento y reparación, vigilancia, etc.), dichas actividades constituyen los Procesos Técnicos a satisfacer las necesidades de bienes y servicios.

El abastecimiento es una función de apoyo al funcionamiento interno de una organización social. Se orienta a suministrar o proporcionar los elementos materiales (bienes) y las actividades complementarias para la operación de dichos elementos, en estado óptimo o garantizado (servicios); ambos, bienes y servicios, se emplean para la producción gubernativa de servicios públicos o de bienes económicos a través de las distintas entidades que constituyen la administración pública.

En consecuencia, de acuerdo a lo planteado por los autores citados y descritos en los párrafos precedentes, la investigación se basará en teorías de:

- Administracióngeneral

- Logística

- Sistema de abastecimiento

- Planeamiento estratégico y

- Administración pública

\section{Los tres sub sistemas y once procesos técnicos del} sistema de abastecimiento

El sistema de abastecimiento tiene tres subsistemas y once procesos técnicos, tal como podemos visualizar en la Figura $\mathrm{N}^{\circ} 01$.

El objeto de los procesos técnicos del sistema de abastecimiento, es normar los procedimientos del sistema, 


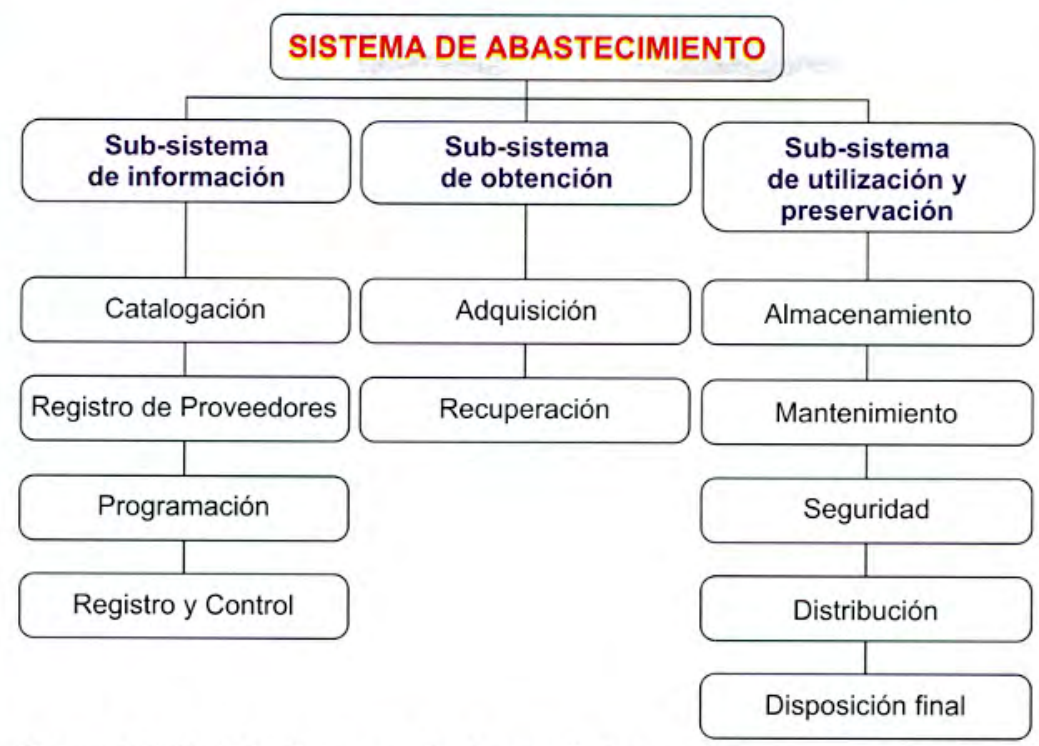

Figura $\mathrm{N}^{\circ} 01$. Sub Sistemas de abastecimiento y sus procesos técnicos Fuente: Elaboración propia

orientados a suministrar y/o abastecer en forma continua, sistemática, racional, oportuna y al costo más económico, los recursos materiales, así como los servicios no personales a los órganos y dependencias de la administración pública, dentro del marco presupuestal, ceñidas a las disposiciones de la Ley del sistema de abastecimiento y otras normas del caso que se encuentran en vigencia.

Asimismo, debemos precisar que la finalidad de la aplicación de los procesos técnicos del sistema de abastecimiento es para asegurar la uniformidad, coordinación, racionalidad y coherencia en los procesos técnicos del sistema para una eficaz y oportuna toma de decisiones por los directivos y funcionarios del organismo público.

\section{Etapas del proceso de abastecimiento}

- Programación

- Adquisición u obtención

- Almacenamiento

- Distribución

Para realizar una programación efectiva, podemos señalar y utilizar las siguientes etapas: (Ver Figura $\mathrm{N}^{\circ} 02$ )

El proceso de programación de abastecimiento requiere de un seguimiento ordenado y metódico en etapas, el órgano de Abastecimiento es el que debe manejar y organizar la información necesaria para formular el plan de obtención.

El proceso de programación de abastecimiento consta de las siguientes fases:

1. Determinación de necesidades

2. Consolidación de necesidades

3. Formulación del plan de obtención

4. Ejecución de la programación

5. Evaluación de la programación

\section{MATERIALES Y MÉTODOS}

Por el tipo de aplicación, la presente investigación es básica, en razón que se utilizan conocimientos de las ciencias administrativas para ser aplicados a nivel de gestión logística.

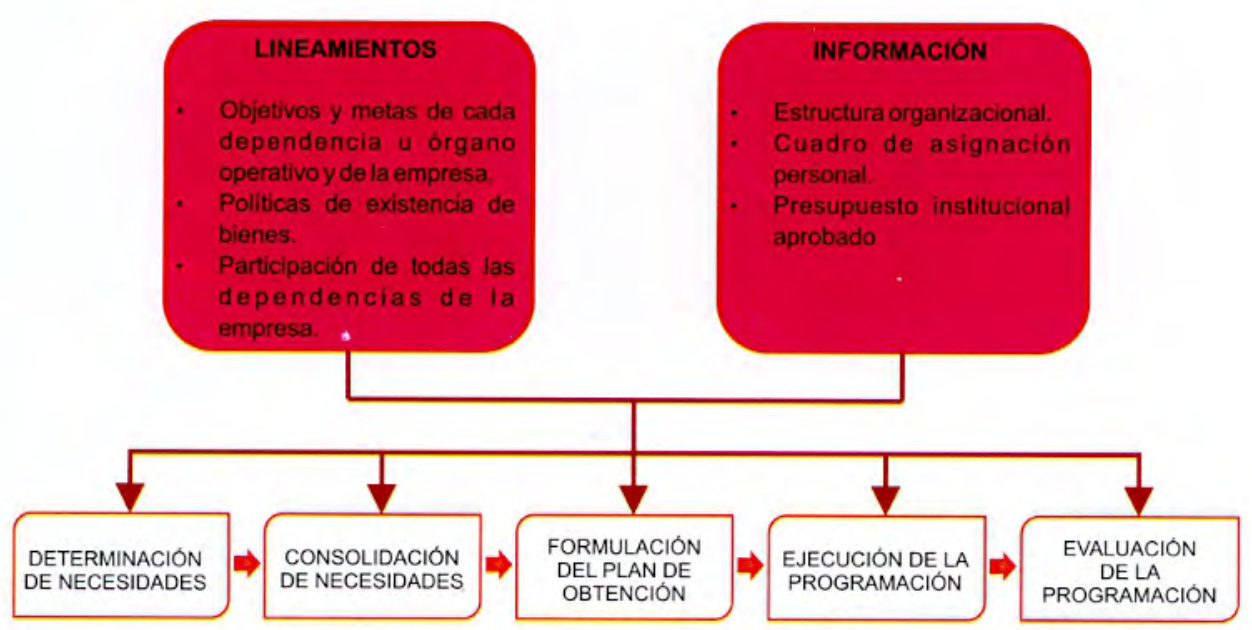

Figura $\mathrm{N}^{\circ} 02$. Programación de abastecimiento

Fuente: Elaboración propia 
En la presente investigación se utilizó un diseño no experimental, descriptivo y correlacional.

No experimental, porque no se pretendió manipular las variables estudiadas sino observarlas tal $y$ como se presentaron en el contexto para analizarlas.

Descriptiva, porque se describe el comportamiento de cada una de las variables involucradas, como son: la programación de abastecimiento y la gestión de logística.

Correlacional, debido a que la investigación se orienta a relacionar las variables involucradas en la presente investigación.

La población que se consideró para efectuar el presente trabajo de investigación al grupo humano que labora en la Oficina de Logística y Servicios de la UNJBG, según se muestra en la siguiente Tabla:

Tabla Nº1. Población objetivo del estudio.

\begin{tabular}{lr}
\multicolumn{1}{c}{ Grupo Ocupacional } & Cantidad \\
\hline Directivo & 01 \\
Funcionarios & 02 \\
Profesionales & 01 \\
Técnicos & 07 \\
Auxiliares & 01 \\
Contrato régimen CAS & 03 \\
Practicantes & 02 \\
& Total \\
\hline
\end{tabular}

Fuente: Oficina de Personal de la UNJBG(2010).

Las técnicas utilizadas en el estudio, fueron: los cuestionarios y el análisis de contenido.

Para el acopio de los datos, se diseñó dos cuestionarios, orientada a obtener información de las variables en estudio, tanto para la programación de abastecimiento y la gestión de logística. Los mismos que fueron dirigidos para los servidores de la Oficina de Logística y Servicios de la UNJBG.

Los cuestionarios parten de identificar las variables mencionadas. A dichas variables se les asignó percepciones y a éstas se les asoció con indicadores que son las preguntas, cuyas respuestas permitieron recolectar información según las categorías o niveles de la escala de Likert.

En este caso, el análisis de contenido se basó en la lectura (textual) de la Ley Decreto Ley $N^{\circ} 22056$ Ley del Sistema Nacional de Abastecimiento, D. Leg. N ${ }^{\circ} 1017$ Ley de Contrataciones del Estado, normas y directivas del Sistema de Logístico. Es necesario puntualizar que la lectura textual a diferencia de la lectura común se realizó siguiendo el método científico, es decir, de manera sistemática, objetiva, replicable y válida, combinando la observación y la producción de los datos y la interpretación o análisis de los datos.

Para el procesamiento de los datos se ha utilizado el software estadístico SPSS versión 18 en español y Microsoft Excel.

\section{RESULTADOSY DISCUSIÓN}

De los resultados obtenidos se puede observar que la gestión de logística en la UNJBG no es eficiente, considerando que no se realiza la programación de abastecimientos.

Cabe referir que la aplicación de la programación de abastecimiento y la gestión de logística, están relacionadas o son dependientes.

Tabla N02. Tabla de contingencia aplicación de la Programación de abastecimiento-gestión logística

\begin{tabular}{lcccc}
\hline $\begin{array}{l}\text { Aplicación de la } \\
\text { Programación de } \\
\text { abastecimiento }\end{array}$ & \multicolumn{3}{c}{ Gestión de la Logística } & Total \\
\hline $\begin{array}{l}\text { Deficiente } \\
\text { Regular }\end{array}$ & 7 & Regular & Eficiente & \\
Eficiente & 1 & 1 & 0 & 10 \\
Total & 0 & 2 & 3 & 5 \\
& 8 & 6 & 0 & 2 \\
\end{tabular}

Fuente: Elaboración propia. SPSS18

Tabla $\mathrm{N}^{\circ} 03$. Pruebas de Chi-Cuadrado

\begin{tabular}{lccc}
\hline & Valor & gl & Sig. Asintótica \\
\hline Chi-cuadrado de Pearson & $12,821^{2}$ & 4 &, 012 \\
Razón de verosimilitudes & 13,245 & 4 &, 010 \\
Asociación lineal por lineal & 4,403 & 1 &, 036 \\
N de casos válidos & 17 & &
\end{tabular}

Fuente: Elaboración Propia. SPSS 18.

a. 9 casillas $(100,0 \%)$ tienen una frecuencia esperada inferior a 5 . La frecuencia minima esperada es, 35 .

\section{CONCLUSIONES}

La programación de abastecimiento incide significativamente en la gestión de logística de la UNJBG. Esta afirmación se ve corroborada en el desarrollo de la investigación, como explicación de los resultados de los cuestionarios desarrollados, para el sustento de la hipótesis general.

Los procesos técnicos de la programación de abastecimiento influyen significativamente en los resultados en la adquisición de bienes y servicios de la UNJBG. Según el resultado del cuestionario se establece que la participación en los procesos de determinación de necesidades, consolidación de necesidades, formulación del plan de obtención, ejecución y evaluación de la programación no se dan a gran escala generando inconvenientes en las adquisiciones de bienes y servicios. De esta manera, la primera hipótesis específica planteada es corroborada.

El nivel de desempeño de la gestión de logística de la UNJBG no es eficiente. El resultado del cuestionario aplicado arroja falta de cumplimiento de las actividades y objetivos de la Oficina de Logística y Servicios de la UNJBG. De esta manera, la segunda hipótesis específica planteada es corroborada.

\section{REFERENCIAS BIBLIOGRÁFICAS}

ANAYA, Julio. (2007), "Innovación y mejora de procesos logísticos". Madrid: ESIC. 
CARTER, Joseph. (2003), "Mejoramiento del proceso de compras". México: Limusa.

CASANOVAS, August. (2003), "Logistica empresarial". Barcelona: Gestión 2000 S.A.

D. Leg. N 1017 (2008), "Ley de Contrataciones del Estado". Lima: Diario Oficial el Peruano.

D.S. No 184-EF (2008), "Reglamento de la Ley de Contrataciones delEstado". Lima: Diario Oficial el Peruano.

GUTIÉRREZ, Gabriel. (1998), 'Logisticay distribución fisica". México: McGraw-Hill.
Kolb, Francois. (1978), "La Logística”. España: Deusto.

Ley $\mathrm{N}^{\circ} 22056$ (1978), "Ley del Sistema Nacional de Abastecimiento". Lima: Diario Oficial el Peruano.

Ley N 29465 (2009), "Ley de Presupuesto del Sector Publico Para el Año Fiscal 2010”. Lima: Diario Oficial el Peruano.

Ley N 29626 (2010), "Ley de Presupuesto del Sector Público Para el Año Fiscal 2011". Lima: Diario Oficial el Peruano.

MARTÍNEZ, Emilio. (2001), "Gestión de compras". México:

Limusa.

\section{Correspondencia:}

Felipe Gómez Cáceres: fgomezc@unjbg.edu.pe
Fecha de Recepción: 10/12/2013

Fecha de Aceptación: 02/07/2014 